Pacific Journal of Mathematics

WEAK INTEGRAL CONVERGENCE THEOREMS AND 


\title{
WEAK INTEGRAL CONVERGENCE THEOREMS AND OPERATOR MEASURES
}

\author{
William V. SMith aNd Don H. TUCKeR
}

\begin{abstract}
An integration theory for vector functions and operator-valued measures is outlined, and it is shown that in the setting of locally convex topological vector spaces, the dominated and bounded convergence theorems are almost equivalent to the countable additivity of the integrating measure. The measures studied are those representing the continuous linear operators on a space of continuous functions. When certain restrictions are imposed on the space involved, actual equivalence of countable additivity and the above theorems obtains, as well as equivalence of certain compactness properties of the operator being represented. An example is given which shows that, in general spaces, convergence in measure no longer implies the almost everywhere convergence of a subsequence.
\end{abstract}

Introduction. One of the more useful and interesting aspects of integrals of scalar-valued functions with respect to scalar-valued measures is that such integrals exhibit certain weakened forms of continuity with respect to integrands, e.g., the dominated convergence theorem, the monotone convergence theorem and the bounded convergence theorem. Professor Angus Taylor once remarked to the second author that one of the detractions from integrals with respect to vector- and operator-valued measures was the unavailability of such convergence theorems.

There are two possible starting points for such theorems; first, where the integrands converge in measure; second, where the integrands converge pointwise almost everywhere. It was shown in [23] that for the vector-valued cases, these types of convergence are not related as they are in the usual scalar case. Examples are given there which show that neither type of convergence implies the other, and an example is given of a scalar-valued sequence which converges pointwise everywhere to 1 and in measure to zero. The examples given there possess pathologies due to the nature of the measure and the measurable sets. However, such pathologies can occur when the measure space is well behaved and the range space for the functions is ill structured. We give an example in this paper of a sequence of vector-valued functions on $[0,1]$ (where the measurable sets are the Lebesgue measurable sets and the measure is Lebesgue measure) which converge in measure but no subsequence converges almost everywhere. 
In [23] it was shown that for the case of convergence in measure, where the measure came from a representation theorem for a continuous linear operator on a space of continuous vector-valued functions with the uniform topology, both a bounded convergence theorem and a dominated convergence theorem hold. In practice, convergence in measure may not be as easily determined as pointwise convergence, thus the second situation seems to be worth careful scrutiny. It is to this case that we direct our attention.

We will show that for a representing measure, countable additivity or continuity of $\mu$ in a given topology implies both a bounded convergence theorem and a dominated convergence theorem in a similar topology. A converse theorem also holds with a change of topologies, and equivalence holds in the case of Banach spaces. As it happens, each of these is also equivalent to a type of weak convergence on the operator which is represented by the measure and thus we are able to classify those operators for which the convergence theorems hold. In general, representing measures are only finitely additive but do exhibit countable additivity in a certain weaker topology (see [10], [11], [25]).

The reason for restricting our attention to representing measures is two-fold. First it allows us to formulate the results in terms of the operators represented; second, there is the technical need to relate the additivity of the measure to the topology of the domain space of the integrands; this second need occurs at an application of the uniqueness of a regular representing measure. If one had begun with such a regular Borel measure which was of bounded semivariation it would indeed have been a representing measure. Thus the use of the term representing measure rather than regular Borel measure of bounded semivariation is a matter of taste.

1. Notation and integration. The representation theory with which we concern ourselves is that developed by R. K. Goodrich in [10] and [11]. We preserve certain facets of the Goodrich notation but do not repeat the development.

In order to state our results in a setting sufficiently general to include a large number of the known integration theorems, we give a brief development of a theory of integration in locally convex topological vector spaces. The resulting integral is not the most general possible, but it suffices for our purposes, namely it allows us to prove the results only once. It is not our intent to intoduce yet another integral for investigation, 
but rather to investigate the problem raised by Taylor for the case of representing measures.

We begin by defining an integral for functions with values in a locally convex topological vector space with respect to an operator-valued measure $\mu$ using a generalization of the methods indicated in Hahn [13] and Vitali [24]. $X$ and $Y$ denote locally convex topological vector spaces, $\{p\}$ and $\{q\}$ separating families of seminorms on $X$ and $Y$, respectively. Let $H$ be a set, $D$ a $\delta$-ring of subsets of $H, \mu$ a function mapping $D$ into $L(X, Y)$, the space of linear operators from $X$ into $Y$ with the property that if $E_{1}$, $E_{2} \in D, E_{1} \cap E_{2}=\varnothing$ and $x \in X$, then $x \mu\left(E_{1} \cup E_{2}\right)=x \mu\left(E_{1}\right)+$ $x \mu\left(E_{2}\right) . L_{c}(X, Y)$ denotes the continuous operators in $L(X, Y)$. An $X$-valued $D$-simple function $f$ is a function of the form

$$
f(h)=\sum_{i=1}^{n} x_{i} \chi_{E_{i}}(h),
$$

where $\chi_{E_{l}}$ is the characteristic function of the set $E_{i}, E_{i} \in D, x_{i} \in X$, for each $i$ and $E_{i} \cap E_{j}=\varnothing(i \neq j)$. Let $S$ be the $\sigma$-ring generated by $D$. Recall that in a $\delta$-ring, if $A \in D$, and $E \in S$, then $A \cap E \in D$ (see Dinculeanu [3, p. 6]). This allows us to define the integral of the $D$-simple function $f$ on a set $E \in S$ as

$$
\sum_{i=1}^{n} x_{i} \mu\left(E_{i} \cap E\right)=\int_{E} f d \mu \in Y .
$$

We assume that for any $q \in\{q\}$ there is a $p \in\{p\}$ such that the following quantity is a finite non-negative number if $E \in S$ :

$$
\sup _{f, D \text {-simple }}\left\{q\left(\int_{E} f d \mu\right) \mid p(f(h)) \leq 1 \text { for all } h \in E\right\}<\infty .
$$

We call this the $q p$-semivariation of $\mu$ on $E$ and write $\hat{\mu}_{q p}(E)$ for this number. A set $E \in S$ will be said to be of measure zero if for all $K \in D$, $K \subseteq E$, we have $\mu(K)=$ the zero operator.

If $m$ is a $Y$-valued set function defined on $S$, then for each $q \in\{q\}$, the $q$-semivariation of $m$ on a set $E \in S$ is defined to be

$$
\begin{aligned}
\sup \left\{q\left(\sum_{i=1}^{k} \alpha_{i} m\left(E_{i} \cap E\right)\right) \mid\left\{E_{i}\right\} \subseteq S ;\left\{\alpha_{i}\right\} \subseteq \mathbf{C} ;\right. \\
\left.\qquad E_{i} \cap E_{j}=\varnothing, i \neq j ;\left|\alpha_{i}\right| \leq 1\right\} .
\end{aligned}
$$


We denote this quantity by $\|m\|_{q}(E)$. For each $q$ and $E$ we have

$$
\sup _{F \subseteq E} q(m(F)) \leq\|m\|_{q}(E) \leq 4 \sup _{F \subseteq E} q(m(F)) .
$$

For each $p \in\{p\}, X_{p}$ will denote the Banach space ( $B$-space) given by the $p$-norm completion of the normed linear space $X / \operatorname{ker}(p)$. The same notation will hold for $Y$.

If $Z$ is a topological space, $B(Z)$ denotes the collection of Borel subsets of $Z$.

Definition 1. $M$ will denote the class of all functions from $H$ into $X$ such that $f$ is strongly measurable in each $X_{p}$. That is, for each $p \in\{p\}$, there exists a sequence of $D$-simple functions $\left\{f_{n p}\right\}$ s.t. $p\left(f_{n p}(h)-f(h)\right)$ $\rightarrow 0$ as $n \rightarrow \infty$ for almost all $h \in H$. The set of measure zero implied by the "almost all" phrase may depend on $p$.

At this point we assume, until otherwise noted, that for all $x \in X$, $x \mu(\cdot)$ is a countably additive $Y$-valued measure, i.e. $\mu$ c.a. (countably additive) will always mean $x \mu(\cdot)$ is c.a. for each $x \in X$ and for some locally convex topology on $Y$. Furthermore we will assume $Y$ is quasicomplete (bounded closed sets in $Y$ are complete), although in many cases we do not require such a strong restriction.

LEMMA 1.2 (An Egoroff Theorem). Let $\left\{f_{n p}\right\}$ denote a sequence of $X$ valued D-simple functions for each $p \in\{p\}$ and suppose that for each $p$, $f_{n p}(h) \rightarrow f(h)$ in $X_{p}$ for all $h \in H$. Define

$$
m_{q}(E)=\sum_{n=1}^{\infty} \frac{\int_{E} f_{n p} d \mu}{\left[1+\sup _{A \in S} q\left(\int f_{n p} d \mu\right)\right] 2^{n}}
$$

for each $E \in S, q \in\{q\}$. Then $m_{q}$ is a countably additive $Y_{q}$-valued measure. For every $q \in\{q\}, p \in\{p\}$ and $E \in S$, there exist sets $N_{q}^{p}$ and $\left\{H_{q_{k}}\right\}_{k=1}^{\infty}$ such that $E / N_{q}^{p}=\cup_{k=1}^{\infty} H_{q_{k}}^{p}$, where $f_{n p} \rightarrow f$ uniformly in $X_{p}$ on $H_{q_{k}}^{p}$ for each $k=(1,2,3, \ldots)$. Furthermore $H_{q_{k}}^{p} \subset H_{q_{k+1}}^{p}, H_{q_{k}}^{p} \in D$ (for each k) and

$$
\left\|\int_{N_{q}^{p}} f_{n p} d \mu\right\|_{q}=0 ; \quad n=1,2, \ldots
$$

Proof. Since $m_{q}$ is a c.a. $Y_{q}$-valued measure, it has a control measure $\lambda_{q}$ (see [5, IV 10.5]). The proof now proceeds as in that found in Halmos $\left[14\right.$, p. 88] using the measure $\lambda_{q}$. 
Lemma 1.3 (A Vitali-Hahn-Saks Theorem). Let $\left\{m_{n}\right\}_{n=1}^{\infty}$ denote a sequence of set functions mapping $S$ into $Y$, and suppose for each $E \in S$, $\lim _{n \rightarrow \infty} m_{n}(E)=m(E)$ exists. If $m_{n}$ is c.a. for each $n$, then so is $m$; the sequence $\left\{m_{n}\right\}$ is uniformly countably additive and converges uniformly to m. Furthermore, for each $q \in\{q\},\left\{\left\|m_{n}\right\|_{q}\right\}_{n=1}^{\infty}$ is a uniformly continuous sequence.

Proof. Consider the $B$-spaces $Y_{q}, q \in\{q\}$. The result now follows from the Vitali-Hahn-Saks Theorem in $B$-spaces (see [5, IV 10.6]).

Definition 1.4. Let $\left\{f_{n}\right\}$ denote a sequence of $D$-simple functions and suppose $f_{n}(h) \rightarrow f(h)$ for each $h \in H$ (then $f \in M$ ). Suppose the integrals $\left\{\int f_{n} d \mu\right\}$ are uniformly c.a. $Y$-valued measures (on $S$ ). Then we say $f \in \mathbf{R}_{s}^{1}$, the first integral class (the $D$-simple functions will be denoted as $R_{s}^{0}$ ), or $f$ is integrable of class 1 . (The $R$ is for F. Riesz, who used a similar definition.)

THEOREM 1.5. For each $f \in \mathrm{R}_{s}^{1}$ there exists a unique countably additive $Y$-valued measure $N_{f}$ such that if $\left\{f_{n}\right\}$ is any sequence satisfying Definition 1.4 then $\lim _{\mu \rightarrow \infty} \int_{E} f_{n} d \mu$ exists uniformly in $E \in S$ and $N_{f}(E)=$ $\lim _{n \rightarrow \infty} \int_{E} f_{n} d \mu$.

Proof. Fix $\left\{f_{n}\right\}$ as in Definition 1.4, $q \in\{q\}$ and $E \in S$. Let $p \in\{p\}$ be such that $\hat{\mu}_{q p}$ is finite on $D$. Apply Lemma 1.2 and notice that for any $D$-simple function $g$,

$$
q\left(\int_{E} g d \mu\right) \leq \sup _{h \in E} p(g(h)) \hat{\mu}_{q p}(E) .
$$

Then with $\left\{f_{n}\right\}, q$ and $p$ prescribed we have

$$
\begin{aligned}
q\left(\int_{E} f_{n}-f_{m} d \mu\right) \leq & q\left(\int_{H_{q k}^{p} \cap E} f_{n}-f_{m} d \mu\right)+q\left(\int_{E \backslash N_{q}^{p} \backslash H_{q k}^{p}} f_{n}-f_{m} d \mu\right) \\
\leq & \sup _{h \in H_{q k}^{p}} p\left(f_{n}(h)-f_{m}(h)\right) \hat{\mu}_{q p}\left(H_{q k}^{p}\right) \\
& +\left\|\int_{E \backslash N_{q}^{p} \backslash H_{q k}^{p}} f_{n} d \mu\right\|_{q}+\left\|\int_{E \backslash N_{q}^{p} \backslash H_{q k}^{p}} f_{m} d \mu\right\|_{q} .
\end{aligned}
$$

The last two terms, for large $k$, are uniformly small in $n$ and $m$, respectively. By the preceding argument and the usual observations (see $\left[15\right.$, p. 80]), we see the limit does not depend on $\left\{f_{n}\right\}$. Application of 
Lemma 1.3 shows the limit exists uniformly in $E \in S$. Finally note that for this argument, $Y$ need only be sequentially complete.

Definition 1.6. Let $f \in \mathbf{R}_{s}^{1}$. If $N_{f}$ is the corresponding measure, we define the indefinite integral of $f$ to be $N_{f}$ and write $\int_{E} f d \mu=N_{f}(E)$ $(E \in S)$.

REMARKS 1.7 (i) If $f_{n} \in \mathrm{R}_{s}^{1}$ and $f_{n}(h) \rightarrow f(h)$ a.e., then $f \in M$ and the conclusion of Lemma 1.2 holds for this sequence.

(ii) It is easily shown that for each $f \in \mathrm{R}_{s}^{1}, q \in\{q\}$ and corresponding $p \in\{p\}$ that

$$
\lim _{\hat{\mu}_{q p}(E) \rightarrow 0}\left\|\int_{E} f d \mu\right\|_{q}=0
$$

(iii) If $f \in \mathbf{R}_{s}^{1}$ then

$$
q\left(\int_{E} f d \mu\right) \leq \sup _{h \in E} p(f(h)) \hat{\mu}_{q p}(E) .
$$

Proof of (iii).

$$
q\left(\int_{E} f d \mu\right) \leq q\left(\int_{E} f_{n}-f d \mu\right)+q\left(\int_{E} f_{n} d \mu\right),
$$

where $\left\{f_{n}\right\}$ is a sequence $\int f d \mu$ and $E \in S$. Fix $p \in\{p\}$ so that $\hat{\mu}_{q p}$ is finite on $E$ and let $\left\{H_{q k}^{p}\right\}$, etc. be as in Lemma 1.2. We then have

$$
\begin{aligned}
q\left(\int_{E} f_{n} d \mu\right) & \leq q\left(\int_{H_{q k}^{p}} f_{n} d \mu\right)+q\left(\int_{E \backslash N^{p} \backslash H_{q k}^{p}} f d \mu\right) \\
& \leq \sup _{h \in H_{q k}^{p}} p\left(f_{n}(h)\right) \hat{\mu}_{q p}\left(H_{q k}^{p}\right)+q\left(\int_{E \backslash N_{q}^{p} \backslash H_{q k}^{p}} f_{n} d \mu\right) .
\end{aligned}
$$

By uniform countable additivity (Definition 1.4) the last term in ( $\ddagger$ ) can be made small (uniformly in $n$ ) by choosing $k$ large enough. By Lemma 1.2 the first term in ( $\ddagger)$ can be made as close to

$$
\sup _{h \in H_{q k}^{p}} p(f(h)) \hat{\mu}_{q p}\left(H_{q k}^{p}\right)
$$

as we like, which is less than or equal to the RHS of $(\dagger)$. The first term on the RHS of $(*)$ can be made as small as we like by choosing $n$ large - this shows $(\dagger)$ holds. 
(iv) By Lemma 1.3 we see that $f \in \mathbf{R}_{s}^{1}$ if and only if there is a sequence $\left\{f_{n}\right\} \subseteq \mathbf{R}_{s}^{0}$ s.t. $f_{n}(h) \rightarrow f(h)$ a.e. and $\lim _{n \rightarrow \infty} \int_{E} f_{n} d \mu$ exists for each $E \in S$.

Definition 1.8. Suppose $\Omega$ is the first uncountable ordinal and $\alpha$ is an ordinal $\alpha<\Omega$. Then either (i) $\gamma$ has an immediate precedessor, $\beta$, or (ii) $\gamma$ is a limit ordinal. In either case define $\mathrm{R}_{s}^{\gamma}$ to be the collection of all functions which are pointwise a.e. limits of sequences in $\cup_{\beta<\gamma} \mathbf{R}_{s}^{\beta}$ such that the limits of the integrals exist (setwise on $S$ ), or, equivalently by Remark 1.7(iv), the integrals of such a sequence are uniformly c.a.

If is clear that the classes $\mathrm{R}_{s}^{\alpha}(\alpha<\Omega)$ are monotone under inclusion and are each contained in $M$.

Definition 1.9. We call a function integrable if it is the pointwise limit (a.e.) of a sequence of functions contained in $\cup_{\alpha<\Omega} \mathbf{R}_{s}^{\alpha}$, the corresponding sequence of integrals converging setwise on $S$. The integral of such a function is defined to be the limit of the sequence of integrals. By our construction, integrable functions are those which lie in one of the classes $\mathrm{R}_{s}^{\alpha}(\alpha<\Omega)$.

REMARKS 1.10. It is well known that in general spaces $X$, the pointwise limit $f$ of a sequence $f_{n}$ of functions which are themselves pointwise limits of simple functions may not be the pointwise limit of a sequence of simple functions. This of course is the reason for Definitions 1.8 and 1.9 (see Thomas [21]). Our construction in Definition 1.8 collapses when $X$ is metrizable. That is, $\mathbf{R}_{s}^{1}=\mathbf{R}_{s}^{\alpha}$ for each $\alpha$. An examination of the previous development shows that if there is a $p \in\{p\}$ s.t. for each $q \in\{q\} \hat{\mu}_{q p}$ is finite on $D$, we may assume $X$ is a subspace of $X_{p}$. Again, our construction collapses (with appropriate restrictions). In fact, if such a $p$ exists, we can relax the definition of $M$ as well. If the range of $\mu$ contains an operator in $L_{c}(X, Y)$ with kernel $=\left\{0_{X}\right\}$ (the additive identity in $X$ ), then the existence of such a $p$ shows that $X$ is a $B$-space with norm $p$. Of course we may add other hypotheses to our assumptions about the functions $\hat{\mu}_{q p}$ - continuity for example. Any of these assumptions will tend to "increase" the set of functions which we can show directly to be integrable. For example, if $H$ is a compact Hausdorff space, with $D=B(H)$ and $f \in C(H)$ (the continuous $X$-valued functions on $H$ ), then if there exists a $p \in\{p\}$ s.t. for each $q \in\{q\} \hat{\mu}_{q p}$ is finite on $D$, or if bounded sets in $X$ are metrizable, or if $X$ has the strict Mackey convergence property (see Gilliam [8]), then $f \in \mathbf{R}_{s}^{1}$ (also see [22]). 
Definition 1.11. A sequence of functions $\left\{f_{n}\right\}$ is said to converge to a function $f$ in semivariation if, for each $K \in D, q \in\{q\}$ and $p \in\{p\}$, such that $\hat{\mu}_{q p}$ is finite on elements of $D$, and for each $\varepsilon>0$, there is an $N \geq 1$ and a set $D(\varepsilon, n, K, q, p) \in D$ such that $n \geq N \Rightarrow \hat{\mu}_{q p}$ $(D(\varepsilon, n, K, q, p))<\varepsilon$, where

$$
D(\varepsilon, n, K, q, p) \supseteq K \cap\left\{h \mid p\left(f_{n}(h)-f(h)\right) \geq \varepsilon\right\} .
$$

Definition 1.11 does not employ our earlier assumption that $x \mu(\cdot)$ is countably additive. If $H \in D$ one can replace $K$ by $H$ and simplify the definition. Furthermore, if $\left\{f_{n}\right\}$ and $f$ are measurable, then the set $\{h \mid$ $\left.p\left(f_{n}(h)-f(h)\right) \geq \varepsilon\right\}$ is in $D$ and one can dispense with the set $D(\varepsilon, n, K, q, p)$, simplifying the definition even more to resemble the usual notion of convergence in measure.

We now give an example which shows that the usual relationship between convergence in measure and pointwise convergence may not hold if $X$ fails to be metrizable.

EXAMPLE. Let $H=[0,1]$, and $S=$ the $\sigma$-algebra of Lebesgue measurable subsets of $[0,1] . \mu$ is Lebesgue measure and $X=\mathbf{R}^{I}$, where $I=[0,1]$ and $\mathbf{R}$ is the set of real numbers with its usual topology. Choose a sequence of real-valued functions converging in measure to some constant $c$, but pointwise nowhere on $H$. Define $\left\{f_{n}\right\}$ as follows: $f_{n}(t)=$ $\left(f_{n}^{i}(t)\right)_{i \in I}$, where $\left\{f_{n}^{0}\right\}$ is the sequence of real-valued functions above. For $i \neq 0$, let $Q=\left\{\left\{n_{k}\right\} \mid\left\{n_{k}\right\} \subseteq N\right.$ (= natural numbers) and $f_{n_{k}}^{0}$ converges a.e. on $H\} . Q$ and $[0,1]$ have the same cardinality, so choose some one-to-one correspondence between them, denoted by $i \leftrightarrow Z$ where $Z \in Q$, $i \in[0,1]$. To define $\left\{f_{n}^{i}\right\}_{n=1}^{\infty}$, let $Z=\left\{n_{k}\right\} \leftrightarrow i, f_{n}^{l}=f_{n}^{0}$ when $n \neq n_{k}$, and, when $n=n_{1}$, let $f_{n_{1}}^{i}=f_{1}^{0}, n=n_{2}, f_{n_{2}}^{i}=f_{2}^{0}$, etc. Then $\left\{f_{n}^{i}\right\}_{n=1}^{\infty}$ converges in measure to $c$ and nowhere on $H$. Furthermore, $\left\{f_{n_{k}}^{l}\right\}$ has the same property when $\left\{n_{k}\right\} \in Q$ and $\left\{n_{k}\right\}=Z \leftrightarrow i$. It follows that $\left\{f_{n}\right\}$ converges in measure, but no subsequence converges a.e.

\section{Main results.}

REMARKS 2.1. We wish to remark that a portion of our results can be stated without reference to linear operators. However, we emphasize that the questions investigated here arise most naturally in the operator-theoretic context. The fact that representing measures are "weakly" c.a. is the whole point of our exercise here. This fact gives our integration theory its utility and allows the proofs to go through in the vector function-operator 
measure case, which is essentially different from the ordinary vector measure case; in fact, our weak c.a. has no genuine analogue there.

Before stating the main theorems we recall some facts about representing measures and other topics. $Y^{\prime}$ is the continuous dual of $Y, Y^{\prime \prime}$ the continuous bi-dual. Let $\sigma\left(Y^{\prime \prime}, Y^{\prime}\right)$ denote the $Y^{\prime}$ topology of $Y^{\prime \prime}$ and let $\left\{q^{\prime}\right\}$ be a family of seminorms generating this topology. Since representing measures can be taken as being defined on $B(H)$ (see [10]), $H$ a compact Hausdorff space, and are $L_{c}^{\prime \prime}(X, Y)$-valued, $x \mu(\cdot)$ being countably additive in the $\left(Y^{\prime \prime} Y^{\prime}\right)$ topology for each $x \in X$, we may define our integral with convergence in $\left\{q^{\prime}\right\}$ rather than $\left\{q^{\prime \prime}\right\}$, where $\left\{q^{\prime \prime}\right\}$ denotes the family of continous seminorms generating the topology on $Y^{\prime \prime}$ sometimes called the " $\varepsilon^{00}$ topology" [20, p. 71], which is the usual norm topology of $Y^{\prime \prime}$ when $Y$ is a normed space. If $\mu$ is such a representing measure, then for each $q^{\prime \prime} \in\left\{q^{\prime \prime}\right\}$ there is a $p^{\prime \prime} \in\left\{p^{\prime \prime}\right\}$ s.t. $\hat{\mu}_{q^{\prime \prime} p^{\prime \prime}}$ is finite on $D$. Of course when $f$ is integrable with respect to a measure countably additive in the $\varepsilon^{00}$ topology, it is integrable in the $\left\{q^{\prime}\right\}$ topology to the same value. For definition of the terms $C^{+}(H), T^{+}, R$, etc. which occur in the statements and proofs of the following theorems, we refer to Goodrich [10] and [11].

In Theorems $2.2-2.4, H$ is a compact $T_{2}$ space and $C(H, X)$ is the space of continuous $X$-valued functions defined on $H$.

THEOREM 2.2. Let $T$ be a continuous linear operator mapping $C(H, X)$ into $Y$, and let $\mu$ be its representing measure defined on $B(H)$. If $x \mu(\cdot)$ is c.a. for each $x \in X$ in the $\varepsilon^{00}$ topology (integration is in the $\varepsilon^{00}$ topology) then:

(1) For each $x \in X$, if $\left\{f_{n}\right\} \subseteq C^{+}(H)$ with $f_{n}(h) \rightarrow f(h)$ a.e. and $\left\|f_{n}\right\|_{\infty} \leq M<\infty$, then $\left\{T^{+}\left(x f_{n}\right)\right\}$ is Cauchy in the $\varepsilon^{00}$ topology.

(2) If $\left\{f_{n}\right\} \subseteq \cup_{\alpha<\Omega} \mathbf{R}_{s}^{\alpha}, f_{n}(h) \rightarrow f(h)$ a.e., and for each $q^{\prime \prime} \in\left\{q^{\prime \prime}\right\}$ there exists an integrable function $g_{q^{\prime \prime}}$ such that for all $E \in B(H)$,

$$
q^{\prime \prime}\left(\int_{E} f_{n} d \mu\right) \leq \sup _{F \leq E} q^{\prime \prime}\left(\int_{F} g_{q^{\prime \prime}} d \mu\right)
$$

then $f$ is integrable, and if $\left\{f_{n}\right\} \subseteq \operatorname{Domain}\left(T^{+}\right)$, then $\left\{T^{+}\left(f_{n}\right)\right\}$ is Cauchy in the $\varepsilon^{00}$ topology.

Proof. For (1) it suffices to deal with the $B$-spaces $Y_{q^{\prime \prime}}^{\prime \prime}\|\cdot\|$ will denote the $q^{\prime \prime}$ norm in $Y_{q^{\prime \prime}}^{\prime \prime} . T^{+}$and $C^{+}(H)$ are defined in [10], where the associated algebra of sets $R$ is defined as well. $Y^{*}$ will denote the continuous dual of $Y_{q^{\prime \prime}}^{\prime \prime}$ 
Fix $x \in X$ and let $f_{n} \geq 0,\left\{f_{n}\right\} \subseteq C^{+}(H)$ satisfying the hypothesis in (1). For each $n$ choose a sequence of simple functions $\left\{g_{n, l}\right\}_{l=1}^{\infty}$ converging pointwise up to $f_{n}$. (It is enough to show (1) holds in the case $f_{n} \geq 0$.)

$$
g_{n}=\sum_{i=1}^{k n} a_{i}^{(n)} \chi_{E_{i}}(n)
$$

For simplicity we suppress the $n$. For any $E \in B(H)$,

$$
\int_{E} x g d \mu=\sum_{i=1}^{k} a_{l} x \mu\left(E \cap E_{i}\right)
$$

Let $y^{*} \in Y^{*}$. Then (assume $y^{*}$ is real and $\left\|y^{*}\right\| \leq 1$ )

$$
\begin{aligned}
\left|y^{*} \sum_{i=1}^{k} a_{l} x \mu\left(E \cap E_{i}\right)\right| & =\left|\sum_{i=1}^{k} y^{*} a_{i} x \mu\left(E \cap E_{l}\right)\right| \\
& =\left|\Sigma_{+} a_{i} y^{*} x \mu\left(E \cap E_{i}\right)+\Sigma_{-} a_{l} y^{*} x \mu\left(E \cap E_{i}\right)\right|
\end{aligned}
$$

(where $\Sigma_{+}$indicates the sum of the positive terms, $\Sigma_{-}$the negative)

$$
\begin{aligned}
& \leq \Sigma_{+} a_{l} y^{*} x \mu\left(E \cap E_{l}\right)-\Sigma_{-} a_{i} y^{*} x \mu\left(E \cap E_{i}\right) \\
& \leq M\left|y^{*}\left(\Sigma_{+} x \mu\left(E \cap E_{l}\right)-\Sigma_{-} x \mu\left(E \cap E_{l}\right)\right)\right| \\
& \leq M\left\|\Sigma_{+}-\Sigma_{-}\right\| \leq M 2 \sup _{F \subseteq E}\|x \mu(F)\| \quad\left(\text { recall } a_{l} \geq 0\right) \\
& \leq 2 M\|x \mu\|(E)
\end{aligned}
$$

$\left(\|x \mu\|\right.$ denotes the $q^{\prime \prime}$-semivariation of $\left.x \mu\right)$.

Therefore

$$
\sup _{F \subseteq E}\left\|\int_{F} x g_{n, l} d \mu\right\| \leq 2 M\|x \mu\|(E)
$$

for all sets $E \in B(H)$. Since $x \mu(\cdot)$ is a c.a. $Y_{q^{\prime \prime}}^{\prime \prime}$-valued measure on the $\sigma$-algebra $B(H)$, it has a control measure $\lambda_{x}$. This implies that the collection of countably additive measures $\left\{\int_{(\cdot)} x g_{n, l} d \mu\right\}_{n, i=1}^{\infty}$ is uniformly c.a. By Theorem 1.5 we have $\lim _{\imath \rightarrow \infty} \int_{F} x g_{n, i} d \mu=\int_{F} x f_{n} d \mu$ uniformly in $F \in B(T)$. Furthermore, $\left\{\int_{(\cdot)} x f_{n} d \mu\right\}$ is a family of uniformly c.a. measures, so, by Theorem 1.5, $\int_{E} x f_{n} d \mu \rightarrow \int_{E} x f d \mu$ as $n \rightarrow \infty$ for each $E \in$ $B(H)$. This concludes the proof of (1). 
For (2), since $\int g_{q^{\prime \prime}} d \mu$ is a c.a. $Y^{\prime \prime}$-valued measure, in the $B$-space $Y_{q^{\prime \prime}}^{\prime \prime}$ it has continuous $q^{\prime \prime}$-semivariation. It follows that the sequence $\left\{\int f_{n} d \mu\right\}$ is uniformly c.a. in $Y_{q^{\prime \prime}}^{\prime \prime}$. By the proof of Theorem 1.5 it is Cauchy. Whenever both the Goodrich integral and our integral exist, they are equal on $H$.

Theorem 2.2 has the following converse. Integration is in the $\sigma\left(Y^{\prime \prime}, Y^{\prime}\right)$ sense.

THEOREM 2.3. Suppose $\mu$ represents the operator $T$ as in Theorem 2.2 and suppose (1) or (2) of Theorem 2.2 holds. Then $x \mu$ is c.a. in the $\varepsilon^{00}$ topology for all $x \in X$.

Proof. If (1) holds choose $\left\{E_{l}\right\}_{i=1}^{\infty} \subseteq R, E_{i} \downarrow \varnothing, x \in X$. The functions $\chi_{E_{1}}$ are bounded (by 1) on $H$ and therefore $\left\{T^{+}\left(x \chi_{E_{t}}\right)\right\}$ is Cauchy in $Y^{\prime \prime}$. But $T^{+}\left(x \chi_{E_{i}}\right)=x \mu\left(E_{i}\right)$. Choose $y^{\prime} \in y^{\prime}$. Then $y^{\prime} x \mu\left(E_{i}\right) \rightarrow 0$ so $\lim _{i \rightarrow \infty} x \mu\left(E_{i}\right)=0_{Y^{\prime \prime}}$. Now select $\left\{E_{i}\right\} \subseteq R, E_{l} \neq \varnothing$ but $\cap E_{l}$ not necessarily in $R$. Then again by $(1), x \mu\left(E_{i}\right) \rightarrow y^{\prime \prime} \in Y^{\prime \prime}$. Therefore by [2, Corollary 18: vii $\Leftrightarrow$ iii] $x \mu(\cdot)$ is strongly bounded, so by the Kluvanek Extension Theorem [17, ix $\Rightarrow \mathrm{i}], x \mu(\cdot)$ has a unique extension to $B(H)$ which is c.a. Of course this must agree with our old $\mu$ since it was c.a. in the $\sigma\left(Y^{\prime \prime}, Y^{\prime}\right)$ topology. If (2) holds, the proof is identical.

Definition 2.4. Suppose $M$ is a topological vector space and $N$ is locally convex with $u$ : $M \rightarrow N$ a linear mapping. $u$ is said to be weakly compact if for some neighborhood $u$ of zero in $M, u(U)$ is relatively compact in the $\sigma\left(N, N^{\prime}\right)$ topology.

If $T: C(H, X) \rightarrow Y$ is linear, we define the operators $T_{x}$ (for $x \in X$ ) on $C(H)$, with its sup norm topology, to $Y$ by

$$
T_{x}(f)=T(x \cdot f) .
$$

THEOREM 2.5. Let $\mu$ be the representing measure for $T$. The following are equivalent:

(i) $T_{x}$ is weakly compact for every $x$.

(ii) $x \mu(E) \in Y$ for all $x$ and $E$.

(iii) $\mu x$ is c.a. in the $\varepsilon^{00}$ topology.

REMARK 2.6. The reader should note that the compactness property in (i) does not imply $T$ is weakly compact on $C(H, X)$. An example illustrating this is given in Dobrakov [4] for Banach spaces. 
Proof. We show (i) $\Rightarrow$ (ii) $\Rightarrow$ (iii) $\Rightarrow$ (i). (i) $\Rightarrow$ (ii). By Lewis [18] there exists a unique measure $m: B(H) \rightarrow Y$ such that $m$ is regular and $T_{x}(f)=$ $\int_{H} f d m$ where the integral is ours. But by Goodrich [11], $x$ is the unique (weakly) regular measure such that $T_{x}(f)=\int f d \mu_{x}$ (we use $d \mu_{x}$ to indicate integration with respect to $x \mu(\cdot))$.

Thus $m(K)=x \mu(K)$ for $K \in R$ by (1) of Theorem 2.2. It follows by the Kluvanek Extension Theorem [17] (as in Theorem 2.3) that $m=x \mu$ on $B(H)$. Thus $x \mu(E) \in Y$ for all $x \in X$ and all $E \in Y$.

(ii) $\Rightarrow$ (iii). This follows immediately from the Grothendieck extension of Pettis' Theorem [12] and the fact that $\mu$ is a representing measure.

(iii) $\Rightarrow$ (i). Let $T_{x}^{* *}$ denote the second adjoint of $T_{x}$. By (1) of Theorem 2.3 we have $T_{x}^{* *}\left(C^{+}(H)\right) \subseteq Y$. Since $Y$ is quasicomplete, Theorem 9.3.1 of Edwards [7] implies $T_{x}$ is weakly compact.

We now define an integral which will be useful in case $x \mu(\cdot)$ is only finitely additive for $x \in X$. We use the technique of Bartle [1]. For now suppose $H \in D$.

Definition 2.7. Let $\left\{f_{n}\right\}$ be a sequence of $D$-simple functions converging in semivariation to a function $f$. Let the integrals of $f_{n}$ be uniformly absolutely continuous, i.e.

$$
\lim _{\hat{\mu}_{q p}(E) \rightarrow 0}\left\|\int_{E} f_{n} d \mu\right\|_{q}=0
$$

(with convergence uniform in $n$ ). We then say $f$ is $B$-integrable with respect to the finitely additive measure $\mu$.

LeMma 2.8. Let $f$ be B-integrable and $\left\{f_{n}\right\}$ as in Definition 2.4. Then $\lim _{n \rightarrow \infty} f_{n} d \mu$ exists for each $E \in D$ and, furthermore, convergence is uniform in $E$. If $\left\{g_{n}\right\}$ is any other sequence as in Definition 2.4, then $\lim \int_{E} g_{n} d \mu=\lim \int_{E} f_{n} d \mu$.

The proof of Lemma 2.8 is similar to that of Bartle [1, Theorem 1].

Definition 2.9. If $f$ is $B$-integrable, and $\left\{f_{n}\right\}$ is as in Definition 7, we write

$$
\int_{E} f d \mu=\lim _{n \rightarrow \infty} \int_{E} f_{n} d \mu
$$

REMARKS. It is easily seen that if $\left\{f_{n}\right\}$ is a sequence of $B$-integrable functions and $\lim _{n \rightarrow \infty} \int_{E} f_{n} d \mu$ converges uniformly in $E$, then $\left\{\int f_{n} d \mu\right\}$ is a uniformly absolutely continuous (with respect to $\mu$ ) sequence of finitely 
additive measures on $D$. Assume $x \mu(\cdot)$ is c.a. for each $x \in X$ and $H \in D$. Then the following proposition holds:

Proposition. If $\left\{f_{n}\right\}$ is a sequence of D-simple functions converging in semi-variation to a function $f$, where some subsequence $\left\{f_{n_{k}}\right\}$ converges to $f$ a.e., then $f$ is $B$ integrable implies $f$ is integrable and the two integrals have the same value in $Y$.

In case $X$ is metrizable, or whenever convergence in semivariation implies pointwise convergence of a subsequence as above, then Theorem 2.2 and 2.3 can be combined by interpreting all integrals which occur as Bartle integrals. The only change required being the modification of statement (2) of Theorem 2.2, dropping the phrase " $f$ is integrable". Conditions (i)-(iii) of Theorem 2.5 would then join in this equivalence as well.

While it is possible to construct integrals which are of a more general type than either of those given in this paper, these usually involve integration of objects other than functions, e.g. see [6] and [23]. Such integrals are useful for many purposes, however they usually sacrifice something in terms of giving information about the objects integrated [19]. Nevertheless, the reader should note that for these more general types of integrals Theorems 2.2 and 2.3 remain valid since our hypotheses restrict the objects to be integrated to functions, and integration in the sense of Definition 1.9 would generally imply integration in the more general cases.

In [6] more general types of representation theorems (in normed spaces) are considered. We note that for these, Theorem 2.2 is easily seen to be true; while in the case of Theorem 2.3 we require some form of countable additivity to be present, e.g. weak* countable additivity on some field of sets whose characteristic functions lie in $F^{+}$(see Theorem 2.2 of [6] for this notation). We can then extend to the smallest $\sigma$-field containing this field as in Theorem 2.3. Ideally one wants that for each $A \in D$, the characteristic function $\chi_{A}$ should lie in $F^{+}$though this is certainly not always true. For the normed case of Goodrich [10] see Uherka [25] and $\S 4$ of [6].

\section{REFERENCES}

[1] R. G. Bartle, A general bi-linear vector integral, Studia Math., 15 (1956), 337-352.

[2] Joe Diestel and Jerry Uhl, Vector Measures, Math Surveys, Amer. Math. Soc., Providence, R.I., 1978.

[3] N. Dinculeanu, Vector Measures, Pergamon, London, 1967. 
[4] I. Dobrakov, On representation of linear operators on $C_{0}(T, X)$, Czechoslovak Math. J., 21 (1971).

[5] N. Dunford and J. Schwartz, Linear Operators. Part I: General Theory, Interscience, New York, 1957.

[6] J. R. Edwards and S. G. Wayment, A unifying representation theorem, Math. Ann., 187 (1970), 317-328.

[7] R. E. Edwards, Functional Analysis, Holt, Rinehart, and Winston, 1965.

[8] D. S. Gilliam, Geometry and the Radon-Nikodym Theorem in strict Mackey convergence spaces, Pacific J. Math., 65 (1976), 353-364.

[9] On integration and the Radon-Nikodym theorem in locally convex topological vector spaces, J. Reine Angew. Math., 292 (1977), 125-137.

[10] R. K. Goodrich, A Riesz representation theorem, Proc. Amer. Math. Soc., 24 (1970), 629-636.

[11] A Riesz representation theorem in the setting of locally convex topological vector spaces, Trans. Amer. Math. Soc., 131 (1968), 256-258.

[12] A. Grothendieck, Sur les applications lineaires faiblement compactes d'espaces du type $C(K)$, Canad. J. Math., 5, 129-173.

[13] H. Hahn, Uber Folgen linearer Operationen, Monatsh Math. Phys., 32 (1922), 3-88.

[14] P. R. Halmos, Measure Theory, Van Nostrand, Princeton, N.J., 1950.

[15] E. Hille and R. S. Phillips, Functional Analysis and Semigroups, Amer. Math. Soc. Colloq. Publ., vol. 31, Providence, R.I., 1957.

[16] I. P. Natanson, Theory of Functions of a Real Variable, Ungar, New York, 1961.

[17] Igor Kluvanek, The Extension and Closure and a Vector Measure, Vector and Operator-Valued Measures and Applications (D. H. Tucker and H. B. Maynard, Editors), Academic Press, New York, 1973, pp. 168-183.

[18] D. R. Lewis, Integration with respect to vector measures, Pacific J. Math., 33, 157-165. (1970).

[19] C. E. Rickart, An abstract Radon-Nikodym theorem, Trans. Amer. Math. Soc., 56 (1944), 50-66.

[20] A. P. Robertson and W. J. Robertson, Topological Vector Spaces, 2nd ed., Cambridge Univ. Press, London, 1973.

[21] G. E. F. Thomas, The Lebesgue-Nikodym theorem for vector valued Radon measures, Mem. Amer. Math. Soc., No. 139 (1974).

[22] Integration of functions with values in locally convex Suslin spaces, Trans. Amer. Math. Soc., 212 (1975), 61-88.

[23] D. H. Tucker and S. G. Wayment, Absolute continuity and the Radon-Nikodym theorem, J. Reine Angew. Math., 244 (1970), 1-19.

[24] G. Vitali, Sull' integrazione per serie, Rend. Circ. Mat. Palermo, 23 (1970), 137-155.

[25] D. J. Uherka, Generalized Stieltjes integrals and a strong representation theorem for continuous linear maps on a function space, Math. Ann., 182 (1969), 60-66.

Received July 31, 1978 and in revised form March 1, 1983.

THE UNIVERSITY OF MISSISSIPPI

UNIVERSITY, MS 38677

AND

UNIVERSITY OF UTAH

Salt Lake City, UT 84112 


\section{PACIFIC JOURNAL OF MATHEMATICS}

EDITORS

Donald BabBITT (Managing Editor)

University of California

Los Angeles, CA 90024

Hugo Rossi

University of Utah

Salt Lake City, UT 84112

C. C. Moore and Arthur Ogus

University of California

Berkeley, CA 94720
J. DugunduI

Department of Mathematics

University of Southern California

Los Angeles, CA 90089-1113

R. FINN and H. SAMELSON

Stanford University

Stanford, CA 94305

\section{ASSOCIATE EDITORS}
R. ARENS
E. F. BECKENBACH
B. H. NeumanN
F. WolF
K. YosHIDA (1906-1982)

\section{SUPPORTING INSTITUTIONS}

UNIVERSITY OF ARIZONA

UNIVERSITY OF BRITISH COLUMBIA

CALIFORNIA INSTITUTE OF TECHNOLOGY

UNIVERSITY OF CALIFORNIA

MONTANA STATE UNIVERSITY

UNIVERSITY OF NEVADA. RENO

NEW MEXICO STATE UNIVERSITY

OREGON STATE UNIVERSITY
UNIVERSITY OF OREGON

UNIVERSITY OF SOUTHERN CALIFORNIA

STANFORD UNIVERSITY

UNIVERSITY OF HAWAII

UNIVERSITY OF TOKYO

UNIVERSITY OF UTAH

WASHINGTON STATE UNIVERSITY

UNIVERSITY OF WASHINGTON 


\section{Pacific Journal of Mathematics}

\section{Vol. 111, No. $1 \quad$ November, 1984}

Harald Brandenburg and Adam Stefan Mysior, For every Hausdorff

space $Y$ there exists a nontrivial Moore space on which all continuous

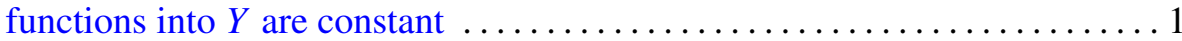

Henry Dappa, A Marcinkiewicz criterion for $L^{p}$-multipliers $\ldots \ldots \ldots \ldots \ldots 9$

P. H. Doyle, III and John Gilbert Hocking, Bijectively related spaces. I.

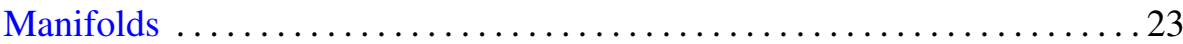

Joel Hass, Complete area minimizing minimal surfaces which are not totally

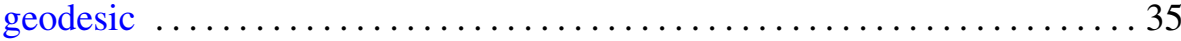

Aarno Hohti, On Ginsburg-Isbell derivatives and ranks of metric spaces .... 39

Richard Howard Hudson, Diophantine determinations of $3^{(p-1) / 8}$ and $5^{(p-1) / 4}$

A. F. Izé and A. Ventura, Asymptotic behavior of a perturbed neutral functional-differential equation related to the solution of the unperturbed

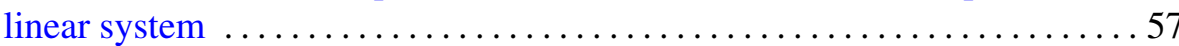

Palle E. T. Jorgensen, Spectral representations of unbounded nonlinear

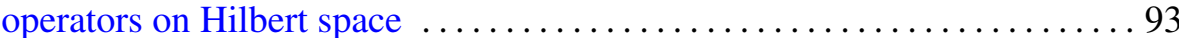

Darrell Conley Kent and Gary Douglas Richardson, Cauchy spaces with

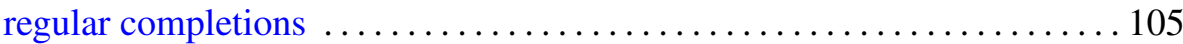

Mark Mahowald, An addendum to: "bo-resolutions" ................ 117

Stuart Wayne Margolis and Jean-Eric Pin, Minimal noncommutative varieties and power varieties

Carla Massaza and Alfio Ragusa, Some conditions on the homology groups of the Koszul complex

Vicente Miquel Molina, Some examples of Riemannian almost-product manifolds

Roderic Murufas, Inverse spectral problems for certain differential operators

Ulrich Oertel, Closed incompressible surfaces in complements of star links

Katsuro Sakai, A characterization of local equiconnectedness

William Victor Smith and Don Harrell Tucker, Weak integral convergence theorems and operator measures 